\title{
A new method for high-order boundary value problems
}

\author{
Yingchao Zhang ${ }^{1 *}$, Liangcai Mei ${ }^{1}$ and Yingzhen Lin ${ }^{1}$
}

${ }^{\text {*Correspondence: }}$

zhych0314@163.com

'School of Applied Science and Civil

Engineering, Zhuhai Campus,

Beijing Institute of Technology,

Zhuhai, China

\begin{abstract}
This paper presents a numerical algorithm for solving high-order BVPs. We introduce the construction method of multiscale orthonormal basis in $W_{2}^{m}[0,1]$. Based on the orthonormal basis, the numerical solution of the boundary value problem is obtained by finding the $\varepsilon$-approximate solution. In addition, the convergence order, stability, and time complexity of the method are discussed theoretically. At last, several numerical experiments show the feasibility of the proposed method.
\end{abstract}

Keywords: Multiscale orthonormal basis; High-order BVPs; Convergence order; Stability

\section{Introduction}

High-order BVPs are important mathematical models in the field of electro-magnetics, fluid mechanics, and material science. Many problems in the theory of elastic stability can be handled by BVPs [1]. It is difficult to find the analytic solutions of high-order BVPs because of the complexity of the systems, many numerical algorithms for high-order BVPs have been proposed in recent years. The multistage integration method is an important method to solve the numerical solution of high-order models by reducing the order gradually [2-5]. Ref. [6-8] discuss the existence of solutions to higher-order differential equations. Cao [9] solved a class of high-order fractional ordinary differential equations by the quadratic interpolation function method. The collocation method proposed by [10] and the orthonormal Bernstein polynomials method proposed by Mirzaee [11, 12] can solve high-order linear complex differential equations effectively. Mirzaee et al. [13-22] proposed a variety of numerical algorithms for solving high-order integro-differential equations. Many scholars have also proposed many methods in the field of numerical solution of high-order partial differential equations [23-25]. Reproducing kernel space is an important Banach space which has been used in the field of numerical analysis. The reproducing kernel methods are used in the numerical solutions of high-order models, singular BVPs, and interface problems [26-31].

In this paper, we construct a set of multiscale orthonormal bases based on the idea of wavelet in the reproducing kernel space. This set of bases is orthonormal, which can improve the computational efficiency. For the numerical solution of differential equations, many literature works, such as [32,33], use the idea of $\varepsilon$-approximate solution. $\varepsilon$ -

(c) The Author(s) 2021. This article is licensed under a Creative Commons Attribution 4.0 International License, which permits use sharing, adaptation, distribution and reproduction in any medium or format, as long as you give appropriate credit to the original author(s) and the source, provide a link to the Creative Commons licence, and indicate if changes were made. The images or other third party material in this article are included in the article's Creative Commons licence, unless indicated otherwise in a credit line to the material. If material is not included in the article's Creative Commons licence and your intended use is not permitted by statutory regulation or exceeds the permitted use, you will need to obtain permission directly from the copyright holder. To view a copy of this licence, visit http://creativecommons.org/licenses/by/4.0/. 
approximate solution provided the stability of the algorithm, good order of convergence in the calculation method. In this article, we construct the multiscale method for the following high-order boundary value problems (BVPs):

$$
\left\{\begin{array}{l}
u^{(m)}+p_{1}(x) u^{(m-1)}+\cdots+p_{m-1}(x) u^{\prime}+p_{m}(x) u=f(x), \quad x \in(0,1) \\
B_{i} u=\alpha_{i}, \quad i=1, \ldots, m,
\end{array}\right.
$$

where $B_{i}(i=1,2, \ldots, m)$ are bounded linear functionals on $W_{2}^{m}[0,1], p_{i}(x)(i=1,2, \ldots, m)$ have certain smoothness.

The paper is organized as follows: In Sect. 2, we construct a set of multiscale orthonormal bases in the reproducing kernel space $W_{2}^{m}[0,1]$. In Sect. 3, we introduce a method to obtain the numerical solution of BVPs by finding an $\varepsilon$-approximate solution, and verify the existence of the $\varepsilon$-approximate solution. In Sect. 4, the convergence, stability, and complexity of this method are discussed. In Sect. 5, we report the numerical result obtained by the present method and compare this method with other previous methods.

\section{Multiscale orthonormal basis}

In this section, the reproducing kernel space is defined and a set of multiscale orthonormal bases is constructed. This knowledge is very useful in the following article.

Definition 2.1 The reproducing kernel space $W_{2}^{m}[0,1]=\left\{u \mid u^{(m-1)} \in C[0,1], u^{(m)} \in\right.$ $\left.L^{2}[0,1]\right\}$, and the inner product of $W_{2}^{m}$ is

$$
\langle u, v\rangle_{W_{2}^{m}}=\sum_{i=0}^{m-1} u^{(i)}(0) v^{(i)}(0)+\int_{0}^{1} u^{(m)} v^{(m)} d x, \quad\|u\|_{W_{2}^{m}}=\sqrt{\langle u, u\rangle_{W_{2}^{m}}} .
$$

Definition 2.2 The reproducing kernel space

$$
W_{2,0}^{m}[0,1]=\left\{u \mid u \in W_{2}^{m}, u(0)=u^{\prime}(0)=\cdots=u^{(m-1)}(0)=0, u^{(m-1)}(1)=0\right\} .
$$

Clearly, $W_{2,0}^{m}[0,1]$ is the closed subspace of $W_{2}^{m}[0,1]$. In Ref. [32], we set up a multiscale orthonormal basis in $W_{2,0}^{1}$ :

$$
\phi_{i, k}(x)=2^{\frac{i-1}{2}} \begin{cases}\left(x-\frac{k}{2^{i-1}}\right), & x \in\left[\frac{k}{2^{i-1}}, \frac{k+1 / 2}{2^{i-1}}\right] \\ \left(\frac{k+1}{2^{i-1}}-x\right), & x \in\left[\frac{k+1 / 2}{2^{i-1}}, \frac{k+1}{2^{i-1}}\right] \\ 0, & \text { else }\end{cases}
$$

where $i=1,2, \ldots, k=0,1,2, \ldots, 2^{i-1}-1$. The graph of $\phi_{i, k}$ is shown in Fig. 1 .

In order to solve Eq. (1.1), this paper constructs a set of orthonormal bases in $W_{2}^{m}[0,1]$ by $\left\{\phi_{i, k}\right\}_{i=1, k=0}^{\infty, 2^{i-1}-1}$. First, let us construct the basis functions in $W_{2,0}^{m}[0,1]$. Note

$$
J_{0}^{m-1} u=\frac{1}{(m-2) !} \int_{0}^{x}(x-s)^{m-2} u(s) d s \quad(m \in N, m \geq 2) .
$$

Theorem $2.1\left\{J_{0}^{m-1} \phi_{1,0}(x), J_{0}^{m-1} \phi_{2,0}(x), J_{0}^{m-1} \phi_{2,1}(x), \ldots, J_{0}^{m-1} \phi_{i, k}(x), \ldots\right\}$ is the multiscale orthonormal basis of $W_{2,0}^{m}[0,1]$. 
Figure $1 \phi_{i, k}(x)$

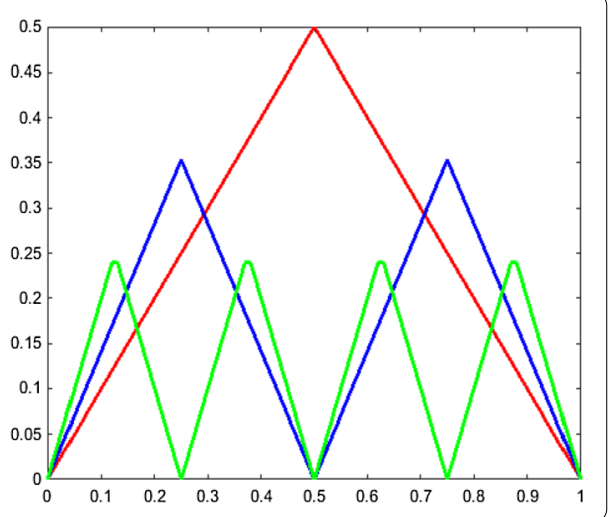

Proof We just prove the orthogonality and completeness.

First, orthonormality. Obviously,

$$
\left\langle J_{0}^{m-1} \phi_{i, k}, J_{0}^{m-1} \phi_{j, m}\right\rangle_{W_{2,0}^{m}}=\left\langle\phi_{i, k}, \phi_{j, m}\right\rangle_{W_{2,0}^{1}}= \begin{cases}1, & i=j, k=m, \\ 0, & \text { else }\end{cases}
$$

orthogonality is true.

Second, completeness. If $\left\langle u, J_{0}^{m-1} \phi_{i, k}\right\rangle_{W_{2,0}^{m}}=0$, then $u \equiv 0$, which means the basis is complete. In fact,

$$
\left\langle J_{0}^{m-1} \phi_{i, k}, u\right\rangle_{W_{2,0}^{m}}=\left\langle\phi_{i, k}, u^{(m-1)}\right\rangle_{W_{2,0}^{1}}=0 \quad \text { implied to } \quad u^{(m-1)}=0
$$

According to Def. 2.2, $u \equiv 0$.

Next, we construct the orthonormal basis in $W_{2}^{m}[0,1]$. There are $m+1$ more conditions in space $W_{2,0}^{m}[0,1]$ than in space $W_{2}^{m}[0,1]$. If the basis in $W_{2}^{m}[0,1]$ is constructed from the basis in $W_{2,0}^{m}[0,1]$, we need to look for $m+1$ functions $g_{k}(x) \in W_{2}^{m}[0,1], k=0,1,2, \ldots, m$, such that

$$
\begin{aligned}
& \left\langle g_{i}(x), g_{j}(x)\right\rangle_{W_{2}^{m}}=0, \\
& \left\langle g_{k}(x), g_{k}(x)\right\rangle_{W_{2}^{m}}=1, \\
& \left\langle g_{i}(x), J_{0}^{m-1} \phi_{i, k}(x)\right\rangle_{W_{2}^{m}}=0 .
\end{aligned}
$$

It is clear that $g_{1}(x)=1, g_{2}(x)=x$ in $W_{2}^{m}[0,1]$ satisfy Eq. (2.2)-Eq. (2.4). Let $g_{k}(x)=a x^{k} \in$ $W_{2}^{m}[0,1],(k=2, \ldots, m)$. By the definition of inner product and Eq. (2.2)-Eq. (2.4), we can obtain $a=\frac{1}{k !}$.

\section{Theorem 2.2}

$$
\left\{\rho_{j}(x)\right\}_{j=1}^{\infty}=\left\{1, x, \frac{x^{2}}{2}, \ldots, \frac{x^{m}}{m !}, J_{0}^{m-1} \phi_{1,0}(x), J_{0}^{m-1} \phi_{2,0}(x), J_{0}^{m-1} \phi_{2,1}(x), \ldots, J_{0}^{m-1} \phi_{i, k}(x), \ldots\right\}
$$

is the multiscale orthonormal basis of $W_{2}^{m}[0,1]$. 
Proof According to Th. 2.1 and Eq. (2.2)-Eq. (2.4), it is clear that

$$
\left\langle\rho_{i}(x), \rho_{j}(x)\right\rangle_{W_{2}^{m}}= \begin{cases}1, & i=j \\ 0, & i \neq j\end{cases}
$$

So $\left\{\rho_{j}(x)\right\}_{j=1}^{\infty}$ is orthogonal.

Next, we just need to prove completeness. That is, if $\left\langle u, \rho_{j}\right\rangle_{W_{2}^{m}}=0$, then $u \equiv 0$. In fact,

$$
\begin{aligned}
& \left\langle u, \frac{x^{k}}{k !}\right\rangle_{W_{2}^{m}}=0 \quad \text { implied to } \quad u^{(k)}(0)=0, \quad k=0,1,2, \ldots, m-1 . \\
& \left\langle u, \frac{x^{m}}{m !}\right\rangle_{W_{2}^{m}}=0 \quad \text { implied to } u^{(m-1)}(1)=0 . \\
& \left\langle u, J_{0}^{m-1} \phi_{i, k}(x)\right\rangle_{W_{2}^{m}}=\left\langle u^{(m-1)}, \phi_{i, k}(x)\right\rangle_{W_{2,0}^{1}}=0 \quad \text { implied to } \quad u^{(m-1)} \equiv 0 .
\end{aligned}
$$

From Eq. (2.5)-Eq. (2.7), $u \equiv 0$.

\section{$3 \varepsilon$-approximate solution of high-order BVPs}

In this section, we give the $\varepsilon$-approximation of Eq. (1.1) and get the numerical solution of BVPs by finding the $\varepsilon$-approximate solution of Eq. (1.1).

Put

$$
L u=u^{(m)}+p_{1}(x) u^{(m-1)}+\cdots+p_{m-1}(x) u^{\prime}+p_{m}(x) u
$$

where $L: W_{2}^{m}[0,1] \rightarrow L^{2}[0,1]$

Theorem 3.1 $L: W_{2}^{m}[0,1] \rightarrow L^{2}[0,1]$ is a bounded linear operator.

Proof Because $W_{2}^{m}[0,1]$ is a reproducing kernel space,

$$
\begin{aligned}
\left|u^{(k)}(x)\right| & =\left|\left\langle u(\cdot), \frac{\partial^{k} K(x, \cdot)}{\partial x^{k}}\right\rangle_{W_{2}^{m}}\right| \\
& \leq\|u(\cdot)\|_{W_{2}^{m}}\left\|\frac{\partial^{k} K(x, \cdot)}{\partial x^{k}}\right\|_{W_{2}^{m}}, \quad k=0,1,2, \ldots, m-1 .
\end{aligned}
$$

By Eq. (3.1), there exist positive constants $M_{k}$ such that

$$
\begin{aligned}
\left\|p_{m-k}(x) u^{(k)}(x)\right\|_{L^{2}} & =\left(\int_{0}^{1}\left(p_{m-k}(x) u^{(k)}(x)\right)^{2} d x\right)^{\frac{1}{2}} \\
& \leq \max _{x \in[0,1]}\left|p_{m-k}(x)\right|\left(\int_{0}^{1}\left(u^{(k)}(x)\right)^{2} d x\right)^{\frac{1}{2}} \\
& \leq M_{k}\|u\|_{W_{2}^{m}}, \quad k=0,1,2, \ldots, m-1 .
\end{aligned}
$$

Therefore

$$
\left\|u^{(m)}\right\|_{L^{2}}^{2}=\int_{0}^{1}\left(u^{(m)}\right)^{2} d x \leq \sum_{i=0}^{2} u^{(i)}(0) u^{(i)}(0)+\int_{0}^{1}\left(u^{(m)}\right)^{2} d x=\|u\|_{W_{2}^{m}}^{2}
$$


From Eq. (3.2) and Eq. (3.3), it follows that

$$
\|L u\|_{L^{2}} \leq M\|u\|_{W_{2}^{m}}
$$

where $M$ is a positive constant.

Then Eq. (1.1) is equivalent to the following equation:

$$
\begin{cases}L u=f(x), & x \in(0,1) \\ B_{i} u=\alpha_{i}, & i=1,2, \ldots, m .\end{cases}
$$

Zhang [32] proposed the $\varepsilon$-approximate theory of second-order differential equations, now we define the $\varepsilon$-approximate solution of Eq. (3.4) based on the idea.

Definition 3.1 $\forall \varepsilon>0, \exists N>0$, when $n>N$, if $\left\|L u_{n}-f\right\|_{L^{2}}^{2}+\sum_{i=1}^{m}\left(B_{i} u_{n}-\alpha_{i}\right)^{2}<\varepsilon^{2}, u_{n}$ is called $\varepsilon$-approximate solution of Eq. (3.4).

Lemma 3.1 $\forall \varepsilon>0, \exists N>0$, when $n>N$,

$$
u_{n}=\sum_{k=1}^{n} c_{k}^{*} \rho_{k}
$$

is the $\varepsilon$-approximate solution of Eq. (3.4), where $c_{k}^{*}$ satisfies

$$
\begin{aligned}
& \left\|\sum_{k=1}^{n} c_{k}^{*} L \rho_{k}-f\right\|_{L^{2}}^{2}+\sum_{l=1}^{m}\left(\sum_{k=1}^{n} c_{k}^{*} B_{l} \rho_{k}-\alpha_{l}\right)^{2} \\
& =\min _{c_{k}}\left[\left\|\sum_{k=1}^{n} c_{k} L \rho_{k}-f\right\|_{L^{2}}^{2}+\sum_{l=1}^{m}\left(\sum_{k=1}^{n} c_{k} B_{l} \rho_{k}-\alpha_{l}\right)^{2}\right] .
\end{aligned}
$$

Put $J$ is a quadratic form about $c=\left(c_{1}, \ldots, c_{n}\right)$,

$$
J\left(c_{1}, \ldots, c_{n}\right)=\left\|\sum_{k=1}^{n} c_{k} L \rho_{k}-f\right\|_{L^{2}}^{2}+\sum_{l=1}^{m}\left(\sum_{k=1}^{n} c_{k} B_{l} \rho_{k}-\alpha_{l}\right)^{2},
$$

$c_{k}^{*}$ is the minimum point of $J$. In fact, in order to find the minimum value of $J$, that is,

$$
\frac{\partial}{\partial c_{j}} J\left(c_{1}, \ldots, c_{n}\right)=0
$$

Because of

$$
\begin{aligned}
& \frac{\partial}{\partial c_{j}} J\left(c_{1}, \ldots, c_{n}\right) \\
& \quad=2 \sum_{k=1}^{n} c_{k}\left\langle L \rho_{k}, L \rho_{j}\right\rangle_{L^{2}}-2\left\langle L \rho_{j}, f\right\rangle_{L^{2}}+2 \sum_{l=1}^{m} \sum_{k=1}^{n} c_{k} B_{l} \rho_{k} B_{l} \rho_{j}-2 \sum_{l=1}^{m} B_{l} \rho_{j} \alpha_{l},
\end{aligned}
$$


then

$$
\sum_{k=1}^{n} c_{k}\left\langle L \rho_{k}, L \rho_{j}\right\rangle_{L^{2}}+\sum_{l=1}^{m} \sum_{k=1}^{n} c_{k} B_{l} \rho_{k} B_{l} \rho_{j}=\left\langle L \rho_{j}, f\right\rangle_{L^{2}}+\sum_{l=1}^{m} B_{l} \rho_{j} \alpha_{l} .
$$

Let

$$
\begin{aligned}
& \mathbf{A}_{\mathbf{n}}=\left(\left\langle L \rho_{k}, L \rho_{j}\right\rangle_{L^{2}}+\sum_{l=1}^{m} B_{l} \rho_{k} B_{l} \rho_{j}\right)_{n \times n}, \\
& \mathbf{b}_{\mathbf{n}}=\left(\left\langle L \rho_{k}, f\right\rangle_{L^{2}}+\sum_{l=1}^{m} B_{l} \rho_{j} \alpha_{l}\right)_{n} .
\end{aligned}
$$

Then Eq. (3.7) changes to

$$
\mathbf{A}_{\mathbf{n}} c=\mathbf{b}_{\mathbf{n}} .
$$

According to [32], the unique solution of Eq. (3.8) is the minimum point of $J$.

\section{Theoretical analysis}

In this section, the properties of the algorithm, such as uniform convergence, stability, and complexity, are introduced.

\subsection{Convergence analysis}

Theorem 4.1 Assume that $u$ is the exact solution of Eq. (1.1), $u_{n}$ is the $\varepsilon$-approximation of Eq. (1.1). If $u^{(m+1)}$ is bounded on [0,1], then $\left|u-u_{n}\right|^{2} \leq 2^{-2 n} C$, where $C$ is a constant.

Proof Assume that

$$
v_{n}(x)=\sum_{j=0}^{m-1} c_{j} \frac{x^{j}}{j !}+\sum_{i=1}^{n} \sum_{k=0}^{2^{i-1}-1} c_{i, k} J_{0}^{2} \phi_{i, k}(x)
$$

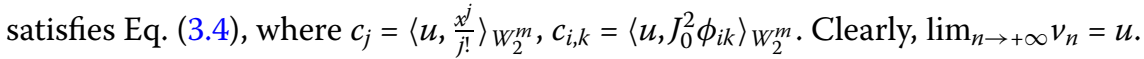

Due to Lemma 3.1, it can get

$$
\begin{aligned}
\left\|u-u_{n}\right\|_{W_{2}^{m}}^{2} & \leq\left\|L^{-1}\right\|^{2}\left\|L u-L u_{n}\right\|_{L^{2}}^{2} \\
& \leq\left\|L^{-1}\right\|^{2}\left(\left\|L u-L u_{n}\right\|_{L^{2}}^{2}+\sum_{i=1}^{m}\left|B_{i} u_{n}-a_{i}\right|^{2}\right) \\
& \leq\left\|L^{-1}\right\|^{2}\left(\left\|L u-L v_{n}\right\|_{L^{2}}^{2}+\sum_{i=1}^{m}\left|B_{i} v_{n}-a_{i}\right|^{2}\right) \\
& \leq\left\|L^{-1}\right\|^{2}\left(\left\|L u-L v_{n}\right\|_{L^{2}}^{2}+\sum_{i=1}^{m}\left|B_{i} v_{n}-B_{i} u\right|^{2}\right) \\
& \leq\left\|L^{-1}\right\|^{2}\left(\|L\|^{2}\left\|u-v_{n}\right\|_{W_{2}^{m}}^{2}+\sum_{i=1}^{m}\left\|B_{i}\right\|^{2}\left\|u-v_{n}\right\|_{3}\right. \\
& \leq M\left\|u-v_{n}\right\|_{W_{2}^{m} .}
\end{aligned}
$$


That is,

$$
\begin{aligned}
\left\|u-u_{n}\right\|_{W_{2}^{m}} & \leq M\left\|u-v_{n}\right\|_{W_{2}^{m}}^{2} \\
& =M\left\|u-\sum_{j=0}^{m} c_{j} \frac{x^{j}}{j !}-\sum_{i=1}^{n} \sum_{k=0}^{2^{i-1}-1} c_{i, k} J_{0}^{2} \phi_{i, k}\right\|_{W_{2}^{m}} \\
& =M \sum_{i=n+1}^{\infty} \sum_{k=0}^{2^{i-1}-1}\left(c_{i, k}\right)^{2} .
\end{aligned}
$$

We can obtain $\left(c_{i, k}\right)^{2} \leq\left(\frac{1}{2}\right)^{3 i} C_{1}$. In fact,

$$
\left|c_{i, k}\right|^{2}=\left|\left\langle u, J_{0}^{m-1} \phi_{i, k}\right\rangle_{W_{2,0}^{m}}\right|^{2}=\left|\int_{0}^{1} u^{(m)}\left(\phi_{i, k}\right)^{\prime} d x\right|^{2}=\left|\int_{0}^{1} u^{(m+1)} \phi_{i, k} d x\right|^{2} .
$$

According to Holder's inequality,

$$
\left|\int_{0}^{1} u^{(m+1)}\left(\phi_{i, k}\right) d x\right|^{2}=\left|\int_{\frac{k}{2^{i-1}}}^{\frac{k+1}{2^{i-1}}} u^{(m+1)}\left(\phi_{i, k}\right) d x\right|^{2} \leq \int_{\frac{k}{2^{i-1}}}^{\frac{k+1}{2^{i-1}}}\left(u^{(m+1)}\right)^{2} d x \int_{\frac{k}{2^{i-1}}}^{\frac{k+1}{2^{i-1}}}\left(\phi_{i, k}\right)^{2} d x .
$$

Because $u^{(m+1)}$ is bound, so $\left|\int_{\frac{k}{2^{i-1}}}^{\frac{k+1}{2^{i-1}}}\left(u^{(m+1)}\right)^{2} d x\right| \leq \frac{1}{2^{i-1}} M$. Then

$$
\begin{aligned}
\left|c_{i, k}\right|^{2} & \leq \frac{1}{2^{i-1}} M \int_{\frac{k}{2^{i-1}}}^{\frac{k+1}{2^{i-1}}}\left(\phi_{i, k}\right)^{2} d x \\
& =M\left(\int_{\frac{k}{2^{i-1}}}^{\frac{k+1 / 2}{2^{i-1}}}\left(x-\frac{k}{2^{i-1}}\right)^{2} d x+\int_{\frac{k}{2^{i-1}}}^{\frac{k+1 / 2}{2^{i-1}}}\left(\frac{k+1}{2^{i-1}}-x\right)^{2} d x\right) \leq\left(\frac{1}{2}\right)^{3 i} C_{1} .
\end{aligned}
$$

So then

$$
\left\|u-u_{n}\right\|_{W_{2}^{m}}^{2} \leq \sum_{i=2 n+1}^{\infty} \sum_{k=0}^{2^{i-1}-1}\left(\left(\frac{1}{2}\right)^{3 i} M\right)=\left(\frac{1}{2}\right)^{2 n} M
$$

where $M$ is a constant.

$$
\left|u(x)-u_{n}(x)\right|^{2}=\left|\left\langle u-u_{n}, K(x, y)\right\rangle_{W_{2}^{m}}\right|^{2} \leq\left(\left\|u-u_{n}\right\|_{W_{2}^{m}}\|K(x, y)\|_{W_{2}^{m}}\right)^{2} \leq 2^{-2 n} C,
$$

where $C$ is a constant, $K(x, y)$ is the reproducing kernel of $W_{2}^{m}$.

From Theorem 4.1, $u_{n}$ uniformly converges to $u$.

\subsection{Stability analysis}

It is well known that if $\mathbf{A}$ is a reversible symmetrical matrix, then the condition number of $\mathbf{A}$ is

$$
\operatorname{cond}(\mathbf{A})_{2}=\left|\frac{\lambda_{1}}{\lambda_{n}}\right|
$$

where $\lambda_{1}$ and $\lambda_{n}$ are the maximum and minimum eigenvalues of $\mathbf{A}$ respectively. 
Obviously, A of Eq. (3.8) is an invertible symmetric matrix. Therefore, in order to prove the stability of the algorithm, we can first prove the boundedness of the eigenvalues.

Lemma 4.1 Suppose $\lambda \mathbf{x}=\mathbf{A x},\|\mathbf{x}\|=1$, where $\mathbf{x}=\left(x_{1}, \ldots, x_{n}\right)^{T}$ is the related eigenvector of $\lambda$, then

$$
C^{2} \leq \lambda \leq\|L\|^{2}+\sum_{l=1}^{m}\left\|B_{l}\right\|^{2}
$$

Proof According to $\lambda \mathbf{x}=\mathbf{A x}$,

$$
\begin{aligned}
& \lambda x_{i}=\sum_{j=1}^{n} a_{i j} x_{j}=\sum_{j=1}^{n}\left(\left\langle L \rho_{i}, L \rho_{j}\right\rangle_{L^{2}}+\sum_{l=1}^{m} B_{l} \rho_{i} B_{l} \rho_{j}\right) x_{j}, \\
& \sum_{j=1}^{n}\left(\left\langle L \rho_{i}, x_{j} L \rho_{j}\right\rangle_{L^{2}}+\sum_{l=1}^{m} B_{l} \rho_{i} x_{j} B_{l} \rho_{j}\right)=\left\langle L \rho_{i}, \sum_{j=1}^{n} x_{j} L \rho_{j}\right\rangle_{L^{2}}+\sum_{l=1}^{m} B_{l} \rho_{i} \sum_{j=1}^{n} x_{j} B_{l} \rho_{j} .
\end{aligned}
$$

Multiply both sides of (4.2) by $x_{i}(i=1,2, \ldots, n)$ and add up to get

$$
\begin{aligned}
\lambda & =\lambda \sum_{j=1}^{n} x_{j}^{2} \\
& =\left\langle\sum_{i=1}^{n} x_{i} L \rho_{i}, \sum_{j=1}^{n} x_{j} L \rho_{j}\right\rangle_{L^{2}}+\sum_{l=1}^{m}\left(\sum_{i=1}^{n} x_{i} B_{l} \rho_{i} \sum_{j=1}^{n} x_{j} B_{l} \rho_{j}\right) \\
& =\left\|\sum_{i=1}^{n} x_{i} L \rho_{i}\right\|_{L^{2}}^{2}+\sum_{l=1}^{m}\left(B_{l} \sum_{j=1}^{n} x_{j} \rho_{i}\right)^{2} \leq\|L\|^{2} \sum_{i=1}^{n} x_{i}^{2}+\sum_{l=1}^{m}\left\|B_{l}\right\|^{2} \sum_{i=1}^{n} x_{i}^{2} \\
& =\|L\|^{2}+\sum_{l=1}^{m}\left\|B_{l}\right\|^{2} .
\end{aligned}
$$

So

$$
\lambda \leq\|L\|^{2}+\sum_{l=1}^{m}\left\|B_{l}\right\|^{2} .
$$

In addition, $\lambda \geq\left\|\sum_{i=1}^{n} x_{i} L \rho_{i}\right\|_{L^{2}}$.

Let $u=\sum_{i=1}^{n} x_{i} \rho_{i}$, then $\lambda \geq\|L u\|_{L^{2}}^{2}$. Without loss of generality, put $\|u\|_{W_{2,0}^{m}}=1$. According to the inverse operator theorem [21], $\|L u\|_{L^{2}} \geq C^{2}\|u\|_{W_{2}^{m}}^{2}$.

So $\lambda \geq\|L u\|_{L^{2}} \geq C^{2}\|u\|_{W_{2}^{m}}^{2}=C^{2}$.

To sum up

$$
C^{2} \leq \lambda \leq\|L\|^{2}+\sum_{l=1}^{m}\left\|B_{l}\right\|^{2}
$$

From Lemma 4.1, we get

$$
\operatorname{cond}(\mathbf{A})_{2}=\left|\frac{\lambda_{1}}{\lambda_{n}}\right| \leq \frac{\|L\|^{2}+\sum_{l=1}^{m}\left\|B_{l}\right\|^{2}}{\frac{1}{C^{2}}}=\left(\|L\|^{2}+\sum_{l=1}^{m}\left\|B_{l}\right\|^{2}\right) C^{2} .
$$

That is, the presented method is stable. 


\subsection{Complexity analysis}

Complexity analysis includes time complexity and space complexity. But ultimately, it is the time efficiency of the algorithm that matters. As long as the algorithm does not take up storage space that is unacceptable to the computer. So this part analyzes the time complexity.

Theorem 4.2 The time complexity of the algorithm is $O\left(n^{3}\right)$.

Proof There are four steps to calculate $\varepsilon$-the approximate solution $u_{n}(x)$ of (3.1).

First, the calculation of matrix $\mathbf{A}_{\mathbf{n}}$ in Eq. (3.8). The matrix $\mathbf{A}_{\mathbf{n}}$ is

$$
\mathbf{A}_{\mathbf{n}}=\left(\left\langle L \rho_{k}, L \rho_{j}\right\rangle+\sum_{l=1}^{m} B_{l} \rho_{k} B_{l} \rho_{j}\right)_{n \times n} .
$$

Set the number of multiplication required to compute $\left\langle L \rho_{k}, L \rho_{j}\right\rangle$ and $B_{l} \rho_{k} B_{l} \rho_{j}$ as $C_{1}, C_{2}$ respectively, $C_{1}, C_{2}$ are constant. So each term of $\mathbf{A}_{\mathbf{n}}$ is evaluated $C_{1}+m C_{2}$ times. Since $\mathbf{A}_{\mathbf{n}}$ is a symmetric matrix, we only need to consider the calculation of the main diagonal and above elements. The first row of $\mathbf{A}_{\mathbf{n}}$ is evaluated $n$ times, the second row $n-1$ times, and so on, so that the total number of multiplications required in calculation of $\mathbf{A}_{\mathbf{n}}$ is

$$
\frac{n(n+1)}{2}\left(C_{1}+m C_{2}\right)
$$

Second, the calculation of vector $\mathbf{b}_{\mathbf{n}}$ in Eq. (3.8). The vector $\mathbf{b}_{\mathbf{n}}$ is

$$
\mathbf{b}_{\mathbf{n}}=\left(\left\langle L \rho_{k}, f\right\rangle+\sum_{l=1}^{m} B_{l} \rho_{j} \alpha_{l}\right)_{n} .
$$

Let the number of multiplication of $\left\langle L \rho_{k}, f\right\rangle$ and $B_{l} \rho_{j} \alpha_{l}$ be $C_{3}, C_{4}, C_{3}, C_{4}$ are constant. So each term of $\mathbf{b}_{\mathbf{n}}$ is evaluated $C_{3}+m C_{4}$ times. So the total number of multiplications required in calculation of $\mathbf{b}_{\mathbf{n}}$ is

$$
\left(C_{3}+m C_{4}\right) n
$$

Third, solve Eq. (3.8). We solve the system by Gaussian elimination. From the mathematical knowledge, Gaussian elimination requires operations

$$
\frac{n(n+1)(2 n+1)}{6}
$$

Forth, calculation $u_{n}$. When calculating the $u_{n}$, the total number of multiplications required is $n$.

To sum up, the total number of multiplication is

$$
\frac{n(n+1)}{2}\left(C_{1}+m\right)+\left(C_{2}+m\right) n+\frac{n(n+1)(2 n+1)}{6}+n=O\left(n^{3}\right) .
$$




\section{Numerical experiments}

In this section, we give several numerical experiments to verify the effectiveness of the proposed algorithm. We denote by $u_{n}(x)$ the approximation to the exact solution $u(x)$ obtained by the numerical schemes in the present work, and we measure the errors in the following sense:

$$
e_{n}(x)=\left|u_{n}(x)-u(x)\right|
$$

where $n$ is the number of bases. C.R. represents the convergence order. All numerical experiments are computed by Mathematica 9.0.

Example 5.1 Ref. [34] mentioned that in order to get the shear deformation of sandwich beams, consider the following third-order BVP:

$$
\left\{\begin{array}{l}
u^{\prime \prime \prime}-k^{2} u^{\prime}+r=0, \quad x \in(0,1) \\
u^{\prime}(0)=u\left(\frac{1}{2}\right)=u^{\prime}(1)=0,
\end{array}\right.
$$

where the physical constants are $k=5$ and $r=1$. The function $u(x)$ shows the shear deformation of sandwich beams. The analytic solution of this problem is

$$
u(x)=\frac{r\left(k(2 x-1) \sinh (k x)+2 \cosh (k x) \tanh \left(\frac{k}{2}\right)\right)}{2 k^{3}} .
$$

The numerical results are given in Table 1.

Example 5.2 Our second example is for fourth-order BVP [31]

$$
\left\{\begin{array}{l}
u^{(4)}-2 u=-1-\left(8 \pi^{4}-1\right) \cos (2 \pi x), \quad x \in(0,1) \\
u(0)=u(1)=u^{\prime}(0)=u^{\prime}(1)=0
\end{array}\right.
$$

Table 2 and Fig. 2 list the error comparison between the multilevel augmentation method [31] and our method for this BVP.

Table 1 Absolute errors of Example 5.1

\begin{tabular}{lllll}
\hline$x$ & Absolute error [28] & Absolute error [29] & Present method $e_{131}(x)$ & Present method $e_{259}(x)$ \\
\hline 0 & $6.65 e-5$ & $2.81 e-5$ & $5.74 e-5$ & $1.34 e-5$ \\
0.1 & $6.50 e-5$ & $2.26 e-5$ & $3.44 e-5$ & $7.65 e-6$ \\
0.2 & $5.25 e-5$ & $1.41 e-5$ & $2.02 e-5$ & $4.08 e-6$ \\
0.3 & $3.63 e-5$ & $7.32 e-6$ & $1.11 e-5$ & $1.81 e-6$ \\
0.4 & $1.87 e-5$ & $2.95 e-6$ & $4.96 e-6$ & $2.55 e-7$ \\
0.6 & $1.73 e-5$ & $2.95 e-6$ & $4.94 e-6$ & $2.23 e-6$ \\
0.7 & $3.40 e-5$ & $7.32 e-6$ & $1.11 e-5$ & $3.80 e-6$ \\
0.8 & $4.98 e-5$ & $1.41 e-5$ & $2.02 e-5$ & $6.09 e-6$ \\
0.9 & $6.20 e-5$ & $2.26 e-5$ & $3.44 e-5$ & $9.68 e-6$ \\
1.0 & $6.34 e-5$ & $2.81 e-5$ & $5.74 e-5$ & $1.54 e-5$ \\
\hline
\end{tabular}


Table 2 Absolute errors of Example 5.2

\begin{tabular}{lllll}
\hline$n$ & Absolute error of [31] & C.R. of [31] & $\max e_{n}(x)$ of present method & C.R. of present method \\
\hline 35 & - & - & $3.693 e-2$ & - \\
67 & $2.004 e-2$ & 1.99 & $9.232 e-3$ & 1.968 \\
131 & $5.013 e-3$ & 2.00 & $2.307 e-3$ & 2.004 \\
259 & $1.253 e-3$ & 1.99 & $5.674 e-4$ & 2.038 \\
515 & $3.133 e-4$ & 1.99 & $1.441 e-4$ & 2.017 \\
\hline
\end{tabular}

Figure 2 The error for Ex. $5.2(n=515)$

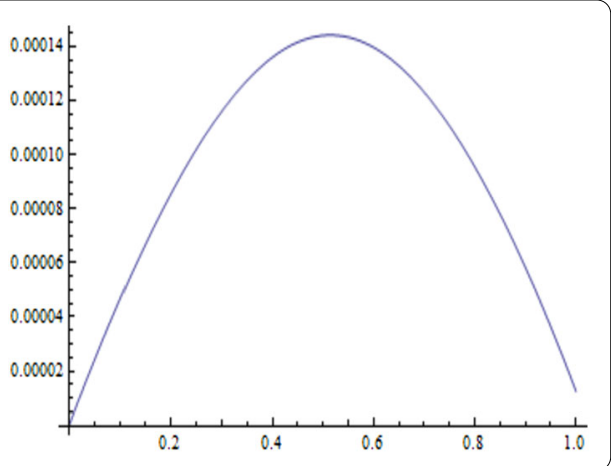

Figure 3 The error for Ex. $5.3(n=67)$

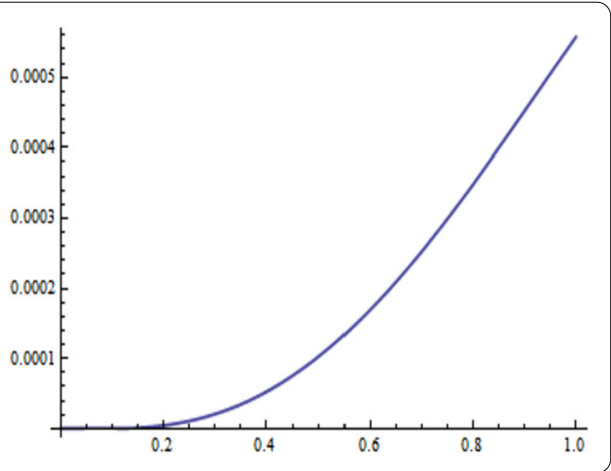

Example 5.3 Our third example is a fifth-order BVP

$$
\left\{\begin{array}{l}
u^{(5)}+u^{(4)}-3 x u^{\prime \prime \prime}-u^{\prime \prime}+u^{\prime}=(2-3 x) e^{x}-1, \quad x \in(0,1) \\
u(0)=u^{\prime}(0)=0, \quad u^{\prime \prime}(0)=1, \\
u^{\prime}(1)=e-1, \quad u^{\prime \prime}(1)=e .
\end{array}\right.
$$

The exact solution of this example is $u(x)=e^{x}-x-1$. Figure 3 shows the error of Example 5.3 .

\section{Conclusion}

In summary, this study used a set of multiscale orthonormal bases to find the $\varepsilon$ approximate solutions of higher-order BVPs. This paper not only demonstrates the convergence and stability in theory, but also demonstrates the feasibility of the method through numerical experiments. Through theoretical analysis and numerical experiments, this method can be extended to solve general linear models, such as linear integral equations, differential equations, and fractional differential equations. 


\section{Acknowledgements}

Not applicable.

\section{Funding}

This work has been supported by the Basic and Applied Basic Research Project of Zhuhai City (ZH22017003200026PWC) and the Characteristic Innovative Scientific Research Project of Guangdong Province (2019KTSCX217).

\section{Availability of data and materials}

The authors declare that all data and materials in this paper are available and veritable.

\section{Competing interests}

The authors declare that they have no competing interests.

\section{Authors' contributions}

This is to declare that all authors have contributed equally and significantly to the contents of the paper. All authors have read and agreed to the published version of the manuscript.

\section{Authors' information}

Zhuhai Campus, Beijing Institute of Technology, Zhuhai, Guangdong, 519085, China

\section{Publisher's Note}

Springer Nature remains neutral with regard to jurisdictional claims in published maps and institutional affiliations.

Received: 28 December 2020 Accepted: 26 April 2021 Published online: 01 May 2021

\section{References}

1. Timoshenko, S.P., Gere, J.M.: Theory of Elastic Stability. McGrawHill-Kogakusha, Tokyo (1961)

2. Jackiewicz, Z., Mittelmann, H.: Construction of IMEX DIMSIMs of high order and stage order. Appl. Numer. Math. 121, 234-248 (2017)

3. Ling, X., Gao, N., Hu, A.: Dynamic analysis of a planetary gear system with multiple nonlinear parameters. J. Comput. Appl. Math. 327, 325-340 (2017)

4. Moradi, A., Sharififi, M., Abdi, A.: Transformed implicit-explicit second derivative diagonally implicit multi stage integration methods with strong stability preserving explicit part. Appl. Numer. Math. 156, 14-31 (2020)

5. Lei, X., Li, J.: Transversal effects of high order numerical schemes for compressible fluid flows. Appl. Math. Mech. 3 343-354 (2019)

6. Lü, X., Cui, M.: Existence and numerical method for nonlinear third-order boundary value problem in the reproducing kernel space. Bound. Value Probl. 2010, 459754 (2010)

7. Ji, Y., Guo, Y., Yao, Y.: Positive solutions for higher order differential equations with integral boundary conditions. Bound. Value Probl. 2015, 214 (2015)

8. Xu, L., Chen, H.: Existence and multiplicity of solutions for fourth-order elliptic equations of Kirchhoff type via genus theory. Bound. Value Probl. 2014, 212 (2014)

9. Cao, J., Wang, Z., Xu, C.: A high-order scheme for fractional ordinary differential equations with the Caputo-Fabrizio derivative. Commun. Appl. Math. Comput. 2(2), 179-199 (2020)

10. Toutounian, F., Tohidi, E., Shateyi, S.: A collocation method based on the Bernoulli operational matrix for solving high-order linear complex differential equations in a rectangular domain. Abstr. Appl. Anal. 4, 215-222 (2013)

11. Mirzaee, F., Samadyar, N., Alipour, S.: Numerical solution of high order linear complex differential equations via complex operational matrix method. SeMA J. 76, 1-13 (2019)

12. Mirzaee, F., et al.: Parameters estimation of HIV infection model of CD4+ T-cells by applying orthonormal Bernstein collocation method. Int. J. Biomath. 11(2), 1850020 (2018)

13. Raslan, K., et al:: Numerical solution of high-order linear integro differential equations with variable coefficients using two proposed schemes for rational Chebyshev functions. New Trends Math. Sci. 4(3), 22-35 (2016)

14. Yzba, S., Yildirim, A., et al.: A collocation approach for solving high-order linear Fredholm-Volterra integro-differential equations. Math. Comput. Model. 55, 547-563 (2012)

15. Mirzaee, F., Rafei, Z:: The block by block method for the numerical solution of the nonlinear two-dimensional Volterra integral equations. J. King Saud Univ., Sci. 23, 191-195 (2011)

16. Mirzaee, F., Hoseini, S.F.: Solving systems of linear Fredholm integro-differential equations with Fibonacci polynomials Ain Shams Eng. J. 5, 271-283 (2014)

17. Mirzaee, F., Hoseini, S.F.: A new collocation approach for solving systems of high-order linear Volterra integro-differential equations with variable coefficients. Appl. Math. Comput. 311, 272-282 (2017)

18. Mirzaee, F., Bimesl, S.: An efficient numerical approach for solving systems of high-order linear Volterra integral equations. Sci. Iran. 21(6), 2250-2263 (2014)

19. Mirzaee, F., Bimesl, S., Tohidi, E.: A numerical framework for solving high-order pantograph delay Volterra integro-differential equations. Kuwait J. Sci. 43(1), 69-83 (2016)

20. Mirzaee, F., Bimesl, S.: Numerical solutions of systems of high-order Fredholm integro-differential equations using Euler polynomials. Appl. Math. Model. 39, 6767-6779 (2015)

21. Mirzaee, F., Bimesl, S.: Application of Euler matrix method for solving linear and a class of nonlinear Fredholm integro-differential equations. Mediterr. J. Math. 11, 999-1018 (2014)

22. Samadyar, N., Orthonormal, M.F.: Bernoulli polynomials collocation approach for solving stochastic Itô-Volterra integral equations of Abel type. Int. J. Numer. Model. 2019, e2688 (2019)

23. Ren, J., Shi, D., Vong, S.: High accuracy error estimates of a Galerkin finite element method for nonlinear time fractional diffusion equation. Numer. Methods Partial Differ. Equ. 36, 284-301 (2020) 
24. Li, Z., Liang, Z., Yan, Y.: High-order numerical methods for solving time fractional partial differential equations. J. Sci. Comput. 71, 785-803 (2017)

25. Mirzaee, F., Bimesl, S.: A new approach to numerical solution of second-order linear hyperbolic partial differential equations arising from physics and engineering. Results Phys. 3, 241-247 (2013)

26. Mei, L.: A novel method for nonlinear impulsive differential equations in broken reproducing kernel space. Acta Math. Sci. 40, $723 C 733(2020)$

27. Zhao, Z., Lin, Y., Niu, J.: Convergence order of the reproducing kernel method for solving boundary value problems. Math. Model. Anal. 21(4), 466-477 (2016)

28. Tirmizi, I.A., Twizell, E.H., Islam, S.U.: A numerical method for third-order non-linear boundary-value problems in engineering. Int. J. Comput. Math. 82, 103-109 (2005)

29. Li, X., Wu, B.: Reproducing kernel method for singular multipoint boundary value problems. Math. Sci. 6(1), 1-5 (2016)

30. Li, X., Wu, B.: A new kernel functions based approach for solving 1-D interface problems. Appl. Math. Comput. 380, $125276(2020)$

31. Chen, Z., Wu, B., Xu, Y.: Multilevel augmentation methods for differential equations. Adv. Comput. Math. 24, 213-238 (2006)

32. Zhang, Y., Sun, H., Jia, Y., Lin, Y.: An algorithm of the boundary value problem based on multiscale orthogonal compact base. Appl. Math. Lett. 101, 106044 (2020)

33. Zheng, Y., Lin, Y., Shen, Y: A new multiscale algorithm for solving second order boundary value problems. Appl. Numer. Math. 156, 528-541 (2020)

34. Haque, M., Baluch, M.H., Mohsen, M.F.N.: Solution of multiple point, nonlinear boundary value problems by method of weighted residuals. Int. J. Comput. Math. 19, 69-84 (1986)

\section{Submit your manuscript to a SpringerOpen ${ }^{\circ}$ journal and benefit from:}

- Convenient online submission

- Rigorous peer review

- Open access: articles freely available online

- High visibility within the field

- Retaining the copyright to your article

Submit your next manuscript at $\boldsymbol{~ s p r i n g e r o p e n . c o m ~}$ 